\section{Cahiers de Narratologie}

Analyse et théorie narratives

$10.1 \mid 2001$

La voix narrative

\title{
L'enfer du langage ou la désorganisation de la lecture dans Le Dernier Soupir du Maure de Salman Rushdie
}

Madelena Gonzalez

\section{CpenEdition}

Journals

Electronic version

URL: http://journals.openedition.org/narratologie/6991

DOI: 10.4000/narratologie.6991

ISSN: 1765-307X

Publisher

LIRCES

\section{Printed version}

Date of publication: 1 January 2001

Number of pages: 521-531

ISBN: 2914561032

ISSN: 0993-8516

Electronic reference

Madelena Gonzalez, "L'enfer du langage ou la désorganisation de la lecture dans Le Dernier Soupir du Maure de Salman Rushdie", Cahiers de Narratologie [Online], 10.1 | 2001, Online since 20 November 2014, connection on 24 February 2021. URL: http://journals.openedition.org/narratologie/6991 ; DOI: https://doi.org/10.4000/narratologie.6991 


\title{
L'ENFER DU LANGAGE OU LA DÉSORGANISATION DE LA LECTURE DANS LE DERNIER SOUPIR DU MAURE DE SALMAN RUSHDIE.
}

\author{
Madelena GONZALEZ \\ Université d'Avignon
}

\section{Un contexte difficile}

Quand il est sorti en septembre 1995 Le Dernier Soupir du Maure fut accueilli avec enthousiasme par la presse et le public comme exploit littéraire dans un climat on ne peut plus hostile à la création artistique. Il a fallu attendre l'apparition d'études critiques spécialisées pour affronter la gêne profonde engendrée par ce roman où l'accès au sens est sans cesse remis en cause. Si le défi à la compréhension présenté par Les Versets sataniques se dénoue finalement dans une parodie élaborée d'épopée, échafaudée à partir d'une structure ternaire bien construite', il semblerait que Le Dernier Soupir du Maure ait érigé en principe la désorganisation de l'écriture dont le résultat est la problématisation de la signification. La littérature de l'exil que revendique sans cesse Rushdie comme source d'inspiration ${ }^{2}$ se double ici de la répression politique et philosophique instaurée par la fatwa. L'écrivain s'en trouve d'autant plus rapproché d'illustres ancêtres et maîtres à penser tels qu'Ovide et Dante, eux aussi exilés, dont les œuvres constituent une partie de la toile intertextuelle du roman. Divine et infernale comédie à la fois, $L e$ Dernier Soupir du Maure affiche un statut oxymorique qui désoriente tout lecteur et dont le ton exalté signalé dans les premiers articles sur le roman ${ }^{3}$ n'est que le versant joyeux

1 Voir à ce propos Milan KUNDERA, Les Testaments trahis et Guy Scarpetta, L'Age d'or du roman.

2 Voir l'essai du même nom dans Patries imaginaires.

3 Voir notamment Tirthankar CHANDA, « Dans le bouillonnement novateur des lettres indo-anglaises ", Le monde diplomatique, juin 
d'une hystérie apocalyptique contraignant le lecteur à errer tel un supplicié à travers les dédales d'une création compromise. Cette œuvre "en noir et du désenchantement ${ }^{4}$ agite cruellement devant le lecteur la promesse de la plénitude et de la félicité éternelle garanties par la présence d'un dieu organisateur du chaos avant de l'enfermer finalement dans un discours auto-destructeur à mesure que le dieu se métamorphose en diable menteur et rapproche les victimes de son imposture du néant de la mort. Ainsi le sommeil du narrateur à la dernière page se mue en interrogation sans concession à l'égard de la fiction et de l'art en général.

\section{Dans les limbes ou le principe de l'incertitude.}

L'écriture postmoderne a familiarisé tout lecteur qui se respecte avec le jeu doux-amer des narrateurs qui jouent les trouble-fêtes et cherchent à le co-opter dans leur non-système où l'incertitude règne. Les premières pages du Dernier Soupir du Maure en fournissent un exemple très frappant ${ }^{5}$. D'emblée le «je » de la narration inquiète plus qu'il ne rassure car en s'auto-identifiant, il formule une pléthore de définitions de soi qui invalident son identité, un paradoxe qui met en abyme le roman lui-même. Un trop plein d'informations crée un vide de sens et un récit à l'envers où nous découvrons la fin avant le début et où la trame narrative ne cesse d'opérer des aller-retour entre des lieux opposés mais qui se ressemblent. Synchronie pour diachronie, voix multiples au lieu de voix unique, lieux instables et changeants ont remplacé «la terre sous nos pieds ${ }^{6}$. Le lecteur, en attente de définitions claires pour pouvoir se situer, erre dans une zone d'incertitude dont les frontières mouvantes ne lui laissent pour toute

1995 ; Peter Kemp, "Absolutely Fabulous ", The Sunday Times, 3 septembre 1995 et Doris Lessing, "Salman Rushdie's Added Spice ", The Sunday telegraph, 3 septembre 1995.

4 Voir à ce propos, Alexis MASSERY and Marc PORÉE, Salman Rushdie, Paris, Editions du Seuil, 1996, pp. 183-204.

5 On se rappellera également le chaos narratif du premier chapitre des Versets sataniques qui suit les deux héros tels des Lucifers contemporains, dans leur chute du ciel vers une terre hostile.

6 Titre, ou presque, du dernier roman de Rushdie (voir notre bibliographie). 
liberté que l'angoisse du non-sens. La perte de repères ${ }^{7}$, un des thèmes moteurs de l'histoire, caractérise donc son écriture et lui confère une indécidabilité chronique où le lecteur navigue péniblement entre choix multiples et variés, balloté d'une histoire à l'autre au gré d'une narration désorganisée et fière de l'être.

Fidèle à sa réputation, le narrateur autodiégétique et biographique est tout sauf fiable, car dans une parodie de la mainmise narrative, il entreprend de miner ses propres affirmations par intervention directe ou en inventant un cadre concurrentiel pour les différentes versions de son ou devraiton dire «ses " histoires. En fait le fonctionnement du texte repose sur la défamiliarisation généralisée car non seulement ses thèmes paraissent excentriques ${ }^{8}$, mais le narrateur symbolise lui-même cette marginalisation de par sa nature hybride et utilise le langage comme support de l'étrangeté : «J'étais (...) un juivolique anonyme, un cathojuif, un fait-tout, un roquet bâtard. J'étais - comment dit-on aujourd'hui ? atomisé. Absolument : un vrai Bombay-mix »9. La compréhension immédiate est compromise, car le texte joue à différer la signification et une insécurité narrative prend le dessus. L'utilisation de tirets par exemple, pour séparer des mots qui devraient former un ensemble compact fait éclater le sens en mosaïque déformante qui prévient toute tentative de classification automatique. De la même manière, expressions exotiques et mots indiens sont jetés dans le chaudron de la phrase rushdienne pour gêner la facilité de compréhension, ce qui rend nécessaire un travail d'interprétation par implication. La narration repose sur des correspondances arbitraires et des radotages du «je » narrant qui semblent faire partie d'un tout artificiel destiné à attirer l'attention sur la nature construite du récit : «Les arbres eux-mêmes sont surpris en plein discours. (N'avez-vous jamais, dans la solitude et le désespoir, parlé aux murs, à votre clébard idiot, à l'air absent ?) Je répète : le

7 Voir MASSERY et PORÉE, op. cit.

8 Il s'agit du récit de la chute d'une dynastie indo-portugaise qui appartient à la minorité catholique du pays et qui se mélange avec une autre communauté encore plus minoritaire, les Juifs de Cochin.

9 Le dernier soupir du maure, p. 125. Toutes les références citées sont tirées de la traduction française de Danielle Marais (1996). 
poivre, s'il vous plaît; car sans le grain de poivre, ce qui s'achève aujourd'hui en Orient et en Occident n'aurait jamais commencé » (p. 17). Les liens, apparemment arbitraires, entre les mots obligent le lecteur à un effort de recul et de distanciation qui vise à relativiser la réalité. Grâce à ce pacte de lecture provocateur qui refuse toute réponse conventionnelle, le lecteur se retrouve spectateur du texte, figé dans les limbes de l'attente permanente d'un sens qui ne cesse de lui échapper. Son engagement envers le texte consistera en une confrontation pénible avec ses contradictions qui fait jaillir sa propre souffrance.

\section{Le voyage vers l'intérieur, le purgatoire comme passage obligé : un examen de conscience douloureux.}

Le voyage vers l'intérieur ${ }^{10}$ des textes postcoloniaux qui affrontent les mythes de la colonisation pour les exorciser devrait permettre au lecteur de Rushdie de se situer sur le terrain familier de la différence culturelle qui récupère son identité à travers le processus d'hybridation qui caractérise l'écriture postcoloniale. Cependant, sous la plume de Rushdie et dans le cas précis du Dernier Soupir du Maure cette identité est sans cesse battue en brèche dans un jeu interminable de fausses pistes. De manière perfide, le narrateur dresse un cadre trompeur pour le récit qui pousse le lecteur à s'ancrer dans une réalité littéraire bien précise avant de le rendre complice de son auto-déconstruction à mesure que la lecture avance. Les nombreux intertextes, La Divine Comédie de Dante, Le Paradis Perdu de Milton, Les Métamorphoses d'Ovide, L'Enéide de Virgile, «Roméo et Juliette ", «Le Marchand de Venise", "Hamlet " et "Othello" de Shakespeare, Les Mille et Une Nuits, divers textes de Kipling et bien d'autres devraient constituer un réseau de pistes significatives qui permettrait de trouver un sens, mais au lieu de ceci ils forment une vaste œuvre de brouillage qui ne fait que déplacer le sens et figurer la difficulté à se situer par rapport à la réalité dont l'intérêt réside dans la réflexion implicite qu'elle suscite à propos de l'instance narrative. Le

10 Cette expression est empruntée à Edward Said, Culture and Imperialism, Londres, Vintage, 1994, pp. 260-1. 
Le Dernier Soupir du Maure de Salman Rushdie

lecteur se voit embarqué dans diverses "galères » génériques ; épopée, conte de fées, fable, roman historique pour se retrouver tel un naufragé, abandonné sur les rives instables d'une œuvre de plus en plus " ouverte » à mesure que la certitude de la catégorisation se dérobe devant lui. Il s'agit pour le lecteur de se soumettre à un jeu sadique de la quête du sens, sauf que ce sens reste insaisissable et l'intérêt du récit se déplace vers l'existence de ce dernier comme processus de création, processus douloureux où le texte, tel un corps souffrant, se dénature pour ne pas rester lettre morte et rend le lecteur complice et compagnon de son supplice. Il se produit un glissement inquiétant de la langue vers une parole de plus en plus désorganisée, personnalisée et arbitraire qui fait de l'oralité du texte un dialogue avec un fou, avec un aliéné dans le vrai sens du terme : celui dont la voix appartient à un autre" ${ }^{11}$. Mais examen de conscience obsessionnel rime avec schizophrénie car la souffrance se double d'un élan jubilatoire et libérateur et attire l'attention sur ses vertus exorcisantes. La vérité résiderait peut-être dans le "cortège surchargé " (p. 102) et l'excès à mesure que la fuite en avant devant le sens se mue en confrontation avec soi-même et fournit une métaphore douloureuse de la lecture : "Dans ma fuite, j'ai fait du monde ma carte personnelle, je l'ai surchargé d'indications, tacheté de $\mathrm{X}$ qui conduisent au trésor de moimême " (p. 16). L'incertitude grandissante fait partie d'une tentative pour se libérer des contraintes de la parole unique et la diabolisation de la voix narrative inscrit dans le texte la déformation comme valeur positive. Comme l'incipit des Versets sataniques nous le rappelle, à travers une citation de L'Histoire du Diable de Daniel Defoe, Satan est apatride et exilé mais jouit aussi d'une certaine liberté et possède des dons créateurs certains. Après tout, n'existe-t-il pas un vieux dicton selon lequel le diable chanterait mieux que Dieu ${ }^{12}$ et le héros ne ressemble-t-il pas à une sirène travestie dont la décadence narrative attire le lecteur non pas vers les berges du Styx mais le rapproche de l'abîme du non-sens ? La

11 Gogol fait partie des influences de Rushdie et nous voyons ici les traces de son Journal d'un fou.

12 Formule que Rushdie reprend à son compte dans Les Versets sataniques, p. 20. 
patience et la persévérance d'un lecteur modèle ne permettront pas à ce dernier d'accéder à la félicité éternelle ou au nirvana du sens mais le contraindront à signer un pacte pervers qui consiste à témoigner de l'auto-dissection du texte et à y participer pour plonger dans les enfers d'une création compromise.

\section{Le royaume du désordre ou l'enfer du langage : «le diable au corps ».}

La clarté illusoire du récit qui tente de passer pour un dialogue sincère avec un lecteur égal tout en le soumettant à une batterie d'affirmations et contre-affirmations contradictoires, attire l'attention sur une polyphonie dérangeante. Le caractère intimiste et rassurant de la convention orale se dégrade en instabilité chronique et le lecteur se perd dans cette tour de Babel monstrueuse qui monte à l'assaut du ciel mais est destinée à s'écrouler dans la confusion. Des formules rassurantes jonchent le texte mais pour mieux révéler le caractère provisoire de la narration, car elles sont minées aussitôt par l'intervention du narrateur, et l'improvisation inspirée de l'oral s'efface devant un impitoyable questionnement métafictionnel où le texte s'épuise à se trouver un sens. En même temps que l'interférence discursive prend le pas sur l'histoire à proprement parler et s'impose comme règle chaotique de la narration, l'humour, l'ironie et le grotesque font irruption dans la trame narrative pour déconstruire la lecture première. En fait le narrateur autobiographique et ses " je " multiples sont à l'image des carreaux de la synagogue de Cochin, reflets d'une réalité complexe, multiple et changeante. Le héros, Moor ou Moraes ${ }^{13}$ n'est que le filtre d'une multitude de voix contradictoires et donc subversives issues du chahut monstrueux d'un royaume infernal et miltonien où le mirage de l'ordre masque le chaos originel et dont la construction satanique parodie l'harmonie du royaume céleste. Ainsi substitués au Dieu tout-puissant de la narration omnisciente, nous découvrons ses fils diaboliques, voix démoniaques et

13 En anglais il s'agit d'une formule protosémantique qui ne passe pas inaperçue : " more he is », c'est-à-dire en français, « il est plus ». 
tentatrices qui nous invitent à nous enfoncer dans les régions souterraines de la déformation. Ayant passé ses portes, gardées non pas par un affreux Cerbère, mais par le chien empaillé du narrateur, symbole ironique de l'impuissance et du néant, le lecteur est condamné à une errance éternelle qui le rapproche de la condition vagabonde du narrateur alors que s'établissent des contre-vérités et contre-créations dignes d'un poète révolté, avatar d'un dieu unique. Car pour Rushdie la voix satanique est surtout la voix de l'autre qui permet de dépasser l'univocité. Habité par une casuistique démente, le texte affirme son pouvoir de manière antinomique et s'autocompromet dans la dissection de soi à l'image de son porte-parole, Moraes, «Je n'étais personne et je ne venais de nulle part, comme un rien, n'appartenant à rien. Voilà qui était mieux, qui sonnait vrai. Tous mes liens s'étaient relâchés. J'avais atteint une anti-Jérusalem : pas une patrie, mais un ailleurs. Un endroit qui ne liait pas mais dissolvait» (p. 441).

Toutefois, l'envers de cette possession diabolique et la perte d'un dieu bienveillant est une autonomie étonnante et la liberté d'un texte constamment remanié qui, par son existence même, ébranle la fixité. La narration ne se fige donc jamais mais se réinvente en permanence comme lieu de transformation du vécu. Dans la mythologie antique, l'Hadès n'est-il pas dépeint comme le lieu des "métamorphoses, des riches gisements, de la germination ${ }^{14}$ et n'affuble-t-on pas Pluton d'une corne d'abondance ?15 Obsédé par les extrêmes, le récit rushdien cherche à pénétrer jusqu'aux frontières de l'exprimable et du concevable en utilisant de manière habile les toiles de l'héroïne du roman, qui est aussi la mère du narrateur, comme mise en abyme d'une réflexion sur le statut de l'art. Corps endomorphe et ectomorphe à la fois, le texte opère un va-et-vient permanent entre le dedans et le dehors ce qui rend son fonctionnement semblable à celui d'une valve du cœur déchaînée où la régulation du flot sanguin n'étant plus assurée, le pouls s'emballe pour instaurer un rythme endiablé qui grise mais met l'organisme en danger. Convié à

14 Jean CHEVALIER et Alain GHEERBRANT, Dictionnaire des symboles, Paris, Robert Lafont, 1992, p. 405.

15 Ibidem. 
ce festin linguistique qui phagocyte l'anglais pour mieux le réinventer, déconcerté par le carnaval métissé de l'imagerie rushdienne, le lecteur est invité à prendre place sur le carrousel déchaîné d'un récit disloqué. Différentes versions d'une réalité instable passent devant ses yeux comme des tableaux vivants et tandis qu'il balance entre banal et merveilleux, bascule du réel au fantastique, se rapproche d'abord pour s'éloigner ensuite, la singularité de la vérité s'efface devant une mosaïque de "milliers de mondes" (p. 102) pour laisser au centre une image distillée qui résume de manière auto-réflexive l'importance du point de vue dans la création du sens. Il s'agit d'un art personnel, subjectif, hybride et surtout construit que le récit rushdien figure par son écriture même, un art qui nécessite un travail de "réimagination ${ }^{16}$ permanente pour ne pas trahir la richesse et la complexité des représentations possibles de la réalité foisonnante de chacun, un art qui se fait une joie de miner ses propres fondements, qui en expose les mécanismes pour mieux les désarticuler et éviter le piège de l'immuabilité mythique. Dans ces conditions, l'œuvre d'art ne peut plus réclamer un statut universel, parlant au nom de tous pour énoncer des vérités éternelles dont tout éloignement sera forcément subversif, mais doit se réinventer comme éphémère, provisoire, composite, «bâtarde », voire satanique ; elle n'est pas taillée dans la pierre ancestrale, mais écrite sur l'air, et va comme elle vient. La gloire mythique du texte, son inventivité monstrueuse sont mises à nu par un narrateur désillusionné pour révéler une dérisoire folie des grandeurs qui avoue ouvertement sa difficulté à signifier. Le lecteur est alors davantage que le témoin résigné de la lente et grotesque agonie de l'énorme baleine échouée qui est l'histoire de Moraes. Tel l'acolyte d'un Satan vagabond, il se débat dans la vase d'un récit moribond, incapable de trouver l'impulsion nécessaire pour se renflouer, crucifié par l'invocation finale du sommeil/mort qui le «cloue » littéralement sur place et fait régner le silence de l'abîme.

16 Ce terme que Rushdie affectionne se traduit difficilement en français car « re-création » semble aussi inexacte que « réinvention ». 
Ainsi le premier et le dernier sens du Dernier Soupir du Maure ne serait-il pas la désolation de l'élégie où l'amour déçu du narrateur, seul système signifiant opposé timidement au chaos, ne nous rapprocherait-il pas logiquement de la mort et ne donnerait-il pas naissance à l'ironie du "RIP " qui couronne la dernière page ? Euvre désespérée et nostalgique de l'humanisme déchu de l'Alhambra de Boabdil, (le souverain éclairé de Grenade), « un dernier soupir pour un monde perdu » (p. 16), le roman de Rushdie dresse contre les limitations du mythe opprimant de la parole sacrée des ayatollahs de la vérité unique, la construction grandiose, personnelle et symbolique d'une liberté absolue révélée dans toute sa souffrance. Pour certains ${ }^{17}$, Rushdie se serait enfermé dans une dialectique stérile et postmoderne de l'hybridité et de la transgression tous azimuts dont le résultat est une certaine décadence idéologique, car elle laisse un vide que les défenseurs de la pensée unique s'empressent de combler ${ }^{18}$. Mais si l'on pourrait arguer que le serpent rushdien se mord donc la queue et que la lecture du Dernier Soupir du Maure ressemble plus à un chemin de croix dénué de salut qu'à une révélation sublime, on pourrait également y opposer la dernière image du roman, celle d'une résurrection possible, qui, avec la récente sortie de La Terre sous ses pieds (Mai 1999), semble indiquer que Rushdie n'a pas renoncé à son univers «édénique-infernal » (p. 28) ni à la conviction que ce dernier pourrait constituer une représentation plus juste de la réalité artistique à une époque où elle est sommée d'épouser l'horreur politique.

17 Notamment Catherine CUNDY, Salman Rushdie, Manchester : Manchester University Press, 1996.

18 Terry EAGLETON formule une critique cinglante des dangers de l'indétermination postmoderne dans son ouvrage Illusions of Postmodernism, Oxford, Blackwell, 1996. 


\section{BIBLIOGRAPHIE}

CHEVALIER (Jean) et GHEERBRANT (Alain) : Dictionnaire des symboles, Paris, Robert Lafont, 1982.

CUNDY (Catherine): Salman Rushdie, Manchester, Manchester University Press, 1996.

EAGLETON (Terry) : Illusions of Postmodernism, Oxford, Blackwell, 1996.

ECO (Umberto) : L'cuvre ouverte, Paris, Le Seuil, 1965.

HENNARD DUTHEIL DE LA ROCHÈRE (Martine) : Origin and Originality in Rushdie's Fiction, Berne, Peter Lang, 1999.

KUNDERA (Milan) : Les testaments trahis, Paris, Gallimard, 1993.

MASSERY (Alexis) and PORÉE (Marc) : Salman Rushdie, Paris, Editions du Seuil, 1996.

RUSHDIE (Salman): The Satanic Verses, Londres, Viking Penguin, 1988.

Traduction française, NASIER (A.) : Paris, Christian Bourgeois, 1989. 1991.

, Imaginary Homelands, Londres, Granta Books,

Traduction française, CHATELIN (Aline) : Paris, Christian Bourgeois, 1993.

Cape, 1995.

, The Moor's Last Sigh, Londres, Jonathan

Traduction française, MARAIS (Danielle) : Paris, Plon, 1996.

The Ground Beneath Her Feet, Londres, Jonathan Cape, 1999.

Traduction française, MARAIS (Danielle) : Paris, Plon, 1999.

SAID (Edward) : Culture and Imperialism, Londres, Vintage, 1994.

SCARPETTA (Guy) : L'âge d'or du roman, Paris, Grasset, 1996. 